\title{
Uma Ferramenta Tridimensional para Apoiar o Ensino de Instalação e Manutenção de Redes de Computadores
}

\author{
Hevander Gabriel Pereira de Souza, Eduardo Filgueiras Damasceno
}

\author{
Universidade Tecnológica Federal do Paraná (UTFPR) - Coordenadoria de Análise e \\ Desenvolvimento de Sistema (COADS) \\ Avenida Alberto Carazzai, 1640 - 86300-000 - Cornélio Procópio, PR - Brasil
}

\begin{abstract}
$\underline{\text { hevander_gabriel@hotmail.com, damasceno@utfpr.edu.br }}$
\end{abstract}
\begin{abstract}
This paper shows a prototype for vocational teaching of cable's manufacturing and connectors for networking devices. Aims to present the tool and propose research on the pedagogical design of this use. Shows a brief review of network simulators systems and its limitation for vocational use.
\end{abstract}

Resumo. Este trabalho descreve um protótipo para o ensino profissional de configuração e instalação de dispositivos de redes de computadores. Tem como objetivo apresentara ferramenta e propor uma investigação sobrea concepção pedagógica deste uso. Mostra uma breve revisão dos sistemas simuladores de rede e sua limitação para o uso ensino técnico e profissionalizante.

\section{Introdução}

O uso de computadores no processo educacional tem como finalidade auxiliar o processo de construção do conhecimento bem como proporcionar meios para que o discente enfrente a sociedade tecnológica presente e futura [Oliveira, 2006].Então, é comumente encontrado diversos aplicativos para o ensino baseado em simulações, seja elas em ambientes computacionais, que abstraem, por meio da computação gráfica, que reproduzem o ambiente real no intuito de apresentar ao aluno uma maneira segura de trabalho e de cooperação [Damasceno e Damasceno, 2013].

A Simulação é o processo de designar um modelo de um sistema real e então conduzir experimentos com este modelo, com o objetivo de ou entender o comportamento do sistema como um todo ou o de avaliar várias estratégias para a operação do sistema [Shannon, 1998]. Desta forma com uma simulação computacional é possível facilitar e melhorar o aprendizado de certo conteúdo ou prática de trabalho promovendo experimentos ou ensaios de ações que em situações reais podem ser arriscadas, demoradas ou onerosas [Adlong, et al, 2003]. Ademais um ambiente de treinamento e simulação deve conter outras características tais como: exploração do ambiente, objetos ou mesmo processos; manipulação em conjunto deste ambiente ou objeto; e reprodutibilidade da ação pedagógica para estudo de caso [Hounsell, Silva \& Gasparini, 2008]. Com o advento da Realidade Virtual (RV) os sistemas tridimensionais ganharam mais potencialidades pelo uso de novos dispositivos de interação [Filippo, et al., 2007] e os ambientes criados se tornaram mais ricos em visualização e simulação, graças as bibliotecas gráficas e de renderização e os mecanismos de física integrados [SAGSET, 2014]. Este trabalho vem apresentar uma ferramenta com visualização tridimensional para treinamento de redes de computadores, com foco nas técnicas relacionadas ao cabeamento estruturado, com o intuito de dar suporte ao processo de treinamento técnico e profissional, subordinando a noções de manutenção e instalação ${ }_{524}$ 


\section{Métodos de Ensino de Instalação de Redes de Computadores}

As mudanças decorrentes das transformações sociais e econômicas no avanço das tecnologias aumenta o apelo do homem está sempre se adaptando e mudando os requisitos de ensino e aprendizagem. Assim, as novas tecnologias abrem espaço para novas formas de ensino, não só na escola, mas também em casa, no trabalho ou em qualquer lugar você pode acessar as informações [Nedic, Machotka\&Nafalski, 2003].

Tendo em conta estes fatores mais importantes na formação das qualidades do técnico de informática é o componente prático de computação currículo. A comunidade de tecnologia da informação profissional esperados técnicos de informática que desenvolveram habilidades práticas durante a sua experiência do curso. Assim, o uso de laboratórios e componentes, práticas com equipamentos adequados para o ensino técnico em informática proporciona aos alunos oportunidades como [Oliveira, 2006]: a) Para testar o conhecimento conceitual; b) Trabalhar de forma colaborativa; c) Para interagir com equipamentos; d) Aprendizagem por tentativa e erro; e) Para a realização da análise de dados experimentais.

No ensino profissionalizante de redes de computadores o aprendizado compreende basicamente duas etapas. A primeira, os alunos aprendem conceitos teóricos expositivas em sala de aula e ter um livro tutorial para orientá-los no estudo e, na segunda etapa, eles executam um conjunto de tarefas em um laboratório, de maneira a alcançar a excelência da prática supervisionada por um docente. No entanto, esse processo tem algumas desvantagens [Martins, 2008]: a) Os tutoriais incluídos em um livro não dão informações suficientes sobre o comportamento dinâmico dos dispositivos de diferentes componentes e novas informações divulgadas pela indústria; b) Os alunos têm conhecimento insuficiente sobre os componentes eletrônicos comerciais, técnicas de montagem e instrumentos; c) Na instalação de componentes os erros são comuns durante as aulas de laboratório; d) Os alunos não possuem conhecimentos teóricos suficientes para entender o "por quê" da operação; e) Os alunos não têm experiência em testes de comportamento de componentes eletrônicos;

Assim, pode-se inferir que se os alunos não têm habilidades ou conhecimentos suficientes de uma estrutura de redes e conexão de computadores, este poderá se ferir, danificar ou perder alguma peça de trabalho por má operação [Damasceno \& Damasceno, 2013]. Então um laboratório virtual poderá fornecer uma maneira de realizar experimentos com os mesmos equipamentos de modo a proteger tanto o aluno quanto os equipamentos físicos disponíveis para o treinamento [Nedic, Machotka\&Nafalski,

2003]. Distinguem-se dois tipos de laboratórios virtuais: (i) de laboratório remoto e (ii) laboratório simulado. Um laboratório remoto é conjunto máquinas e equipamentos monitorados e acompanhados por meio de sensoriamento do laboratório. Já em um laboratório simulado tem um ambiente virtual que simula tanto a operação quanto o estado do equipamento ou do elemento, representado as mesmas ações física, química e eletromagnética encontrado no elemento real.

\section{Ferramentas utilizadas no Ensino de Redes de Computadores}

No desenvolvimento da práxis de ensino de redes de computadores são encontradas algumas intercorrências como, por exemplo: o material didático (a dificuldade para a produção de textos e outros recursos didáticos) e a pouca disponibilidade de equipamentos adequados para a utilização de ferramentas [Ferreira, et al., 2013].

Acredita-se que este fato é devido à velocidade em que os avanços tecnológicos acontecem e ao custo de manutenção de um laboratório experimental. 
Ademais, o ensino de redes de computadores ainda se apresenta de forma bastante tradicional, influenciado diretamente pela forma de organização das principais bibliografias da área [Cantú, 2005]. Esta abordagem, comumente encontrada nos cursos de nível superior contemplam em profundidade a teoria de redes de computadores e sua concepção lógica desempenhada na configuração e no relacionamento entre os protocolos e os dispositivos de interconexão. Toda a expectativa de iniciar um curso de redes de computadores é sufocada no momento em que se mergulha no estudo de padrões e especificidades muitas vezes longe de serem vislumbradas no nível aplicativo.

Uma das abordagens mais repetidas no ensino de redes de computadores é a aplicação da ferramenta Cisco PacketTracer [NetAcad, 2014] que fornece uma simulação, visualização, criação, avaliação e recursos de colaboração e facilita o ensino e aprendizagem de conceitos teóricos mais complexos. Este aplicativo possui uma interface intuitiva e tem o foco no aprendizado de interconexão de dispositivos da fabricante CISCO. Bem como outra aplicação disponível em livre domínio é a NS-2 (Network Simulator, NS-2) [Borin\& Fonseca, 2008], que pode ser definido como um simulador de eventos discretos, focado para o desenvolvimento de pesquisas em redes de computadores o qual e permite a simulação de grande parte dos protocolos utilizados na internet, em redes cabeadas e sem fio.

Há simuladores especializados em pesquisa em redes e interconexão de computadores de uma maneira mais visual, a exemplo os simuladores RouterSim e OPNet Molder, que são comumente usados em cursos de tecnologia em redes de computadores para estudar as redes de comunicação, dispositivos, protocolos e aplicações [Pan, 2010]. Ambos oferecem relativamente um apoio visual que facilita a identificação dos dispositivos e a interface do editor gráfico pode ser usado para construir a topologia e as entidades da camada de aplicativo de rede para uma camada física.

Em alguns experimentos foi detectada a preocupação com a ambientação do aluno enquanto participa de uma instrução por meio do ambiente virtual, que tende a fornecer uma maior abstração do local de aplicação ou trabalho, todavia em casos que não é disponível um laboratório real de mesmo arranjo e espaço físico do que o virtual, verifica-se um desprendimento do aluno e uma baixa motivação ao realizar alguns exercícios virtuais.

Com isso a RV tem obtido grande vazão na pesquisa e aplicação de ambientes simulados para conexão e configuração lógica de redes de computadores. Todavia estas aplicações estão apoiadas apenas no uso sistemático da configuração da camada lógica da rede e de seus protocolos de comunicação de dados. De forma análoga, a utilização de realidade aumentada tem trazido auxílios significativos para a promoção do aprendizado.

De forma a contemplar uma face do curso de redes destinada ao nível de construção e manutenção de instalações e cabeamento, foco principal na formação do profissional técnico, requerido de acordo com o programa PRONATEC [Brasil Pronatec, 2013] este trabalho apresenta um aplicativo simulador que será descrito na próxima sessão.

\section{Apresentação do Protótipo}

Para a construção do sistema objeto deste estudo foi desenvolvido um protótipo do simulador utilizando a técnica de prototipação rápida de ambientes virtuais [Damasceno, et al., 2008], em linguagem RAD (Rapid Application Development), Embarcadero Delphi, com uma API(Application Programming Interface) OpenGL para rápida 
visualização tridimensional. Esta escolha deve-se ao fato da linguagem ser Orientada a Objetos (OO), e os componentes OpenGL na biblioteca GLScene fornecerem uma estrutura de componentes visuais e objetos permitindo a descrição (grafo de cena) e renderização das cenas 3D de modo mais simplificado (esconde as particularidades do OpenGL) e rápido (renderização especializada em tempo real).

O simulador possui algumas fases que serão apresentadas nas próximas figuras deste texto, sendo que há um fluxo didático escolhido e visualizado na Figura 1 é usado pela maioria das escolas técnicas no ensino desta matéria.

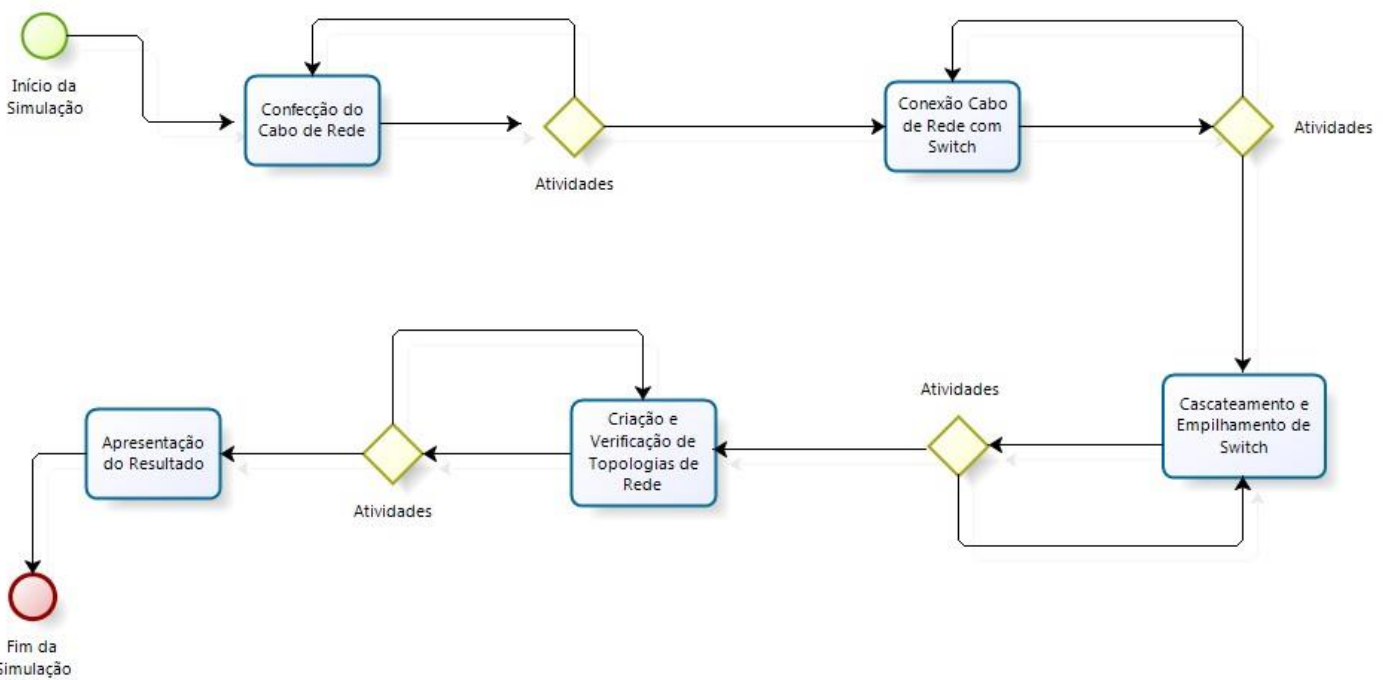

Figura 1. Fluxograma Didático da Aplicação do Simulador

Nas figuras a seguir, estão evidenciadas as telas de cada fase do simulador, no intuito de demonstrar para o leitor a composição do software e sua utilidade no ensino profissionalizante.

A Figura 2a mostra a confecção de um cabo de rede par trançado. Já a figura $2 b$, mostra a conexão de diversos cabos de rede ao dispositivo switch. O usuário pode visualizar de qualquer ângulo todos os componentes podendo aumentar ou diminuir o zoom da ferramenta. A Figura $2 \mathrm{c}$ e $2 \mathrm{~d}$ representa a parte relacionada ao cascateamento e empilhamento de switchs. A figura $2 \mathrm{e}, 2 \mathrm{f}$ e $2 \mathrm{~g}$ mostra o equipamento roteador, com o qual podem ser realizadas as conexões com os cabos criados na figura $2 \mathrm{a}$ com os hosts da cena e verificar a topologia de rede criada. E na Figura $2 \mathrm{~h}$ o resultado das ações de acertos e erros no simulador por usuário.

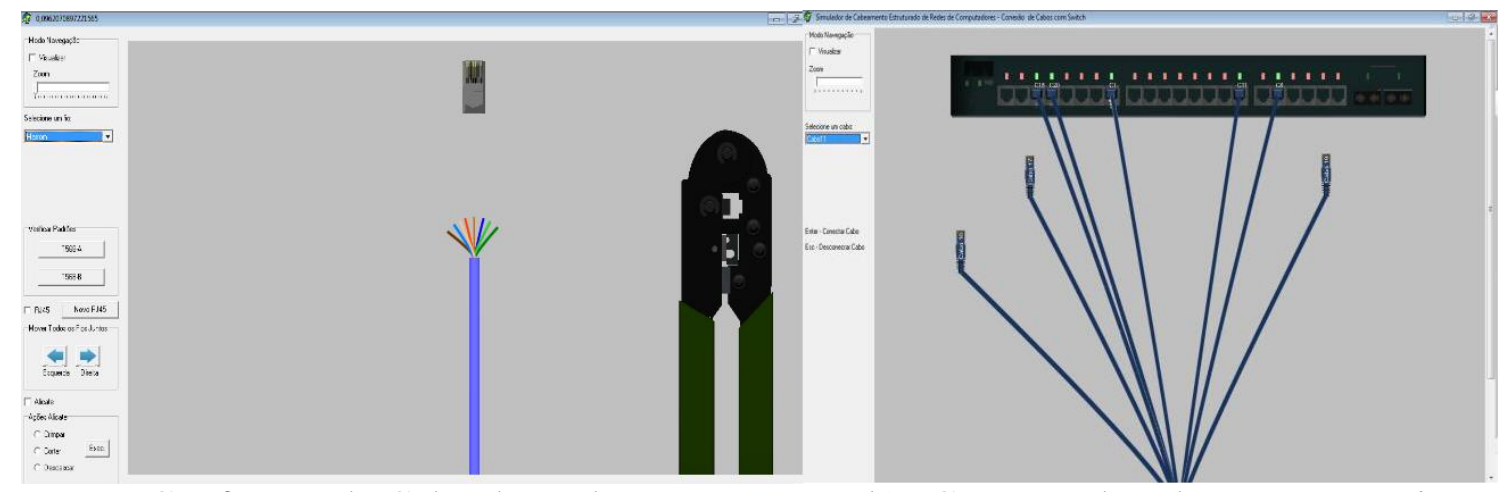

a) Confecção de Cabo de Rede

b) Conexão de cabos com switch 


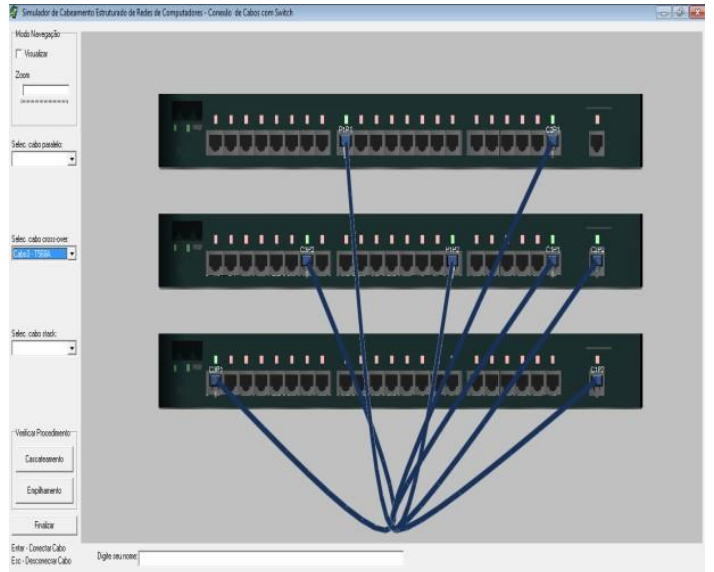

c) Cascateamento de Switchs

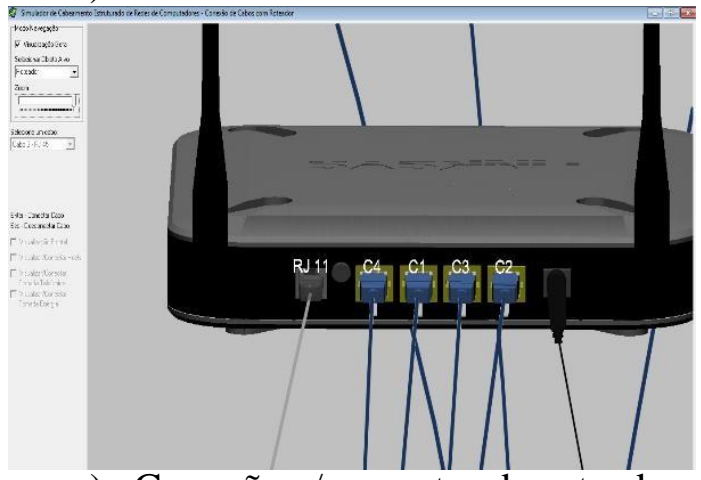

e) Conexão c/ as portas do roteador

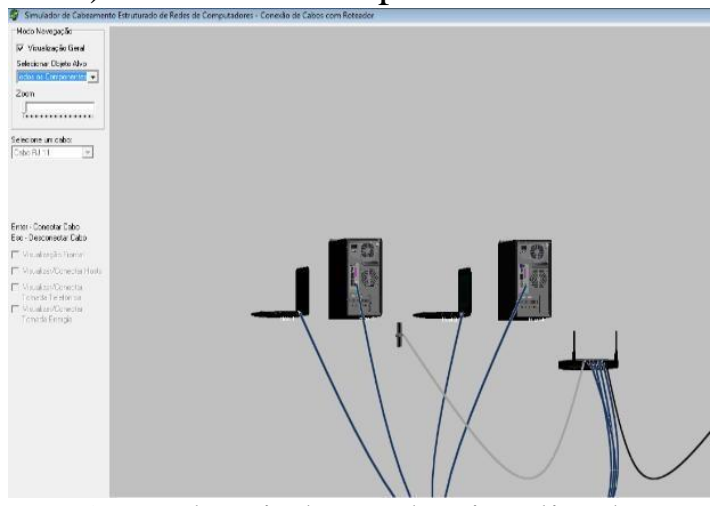

g) Rede criada sendo visualizada

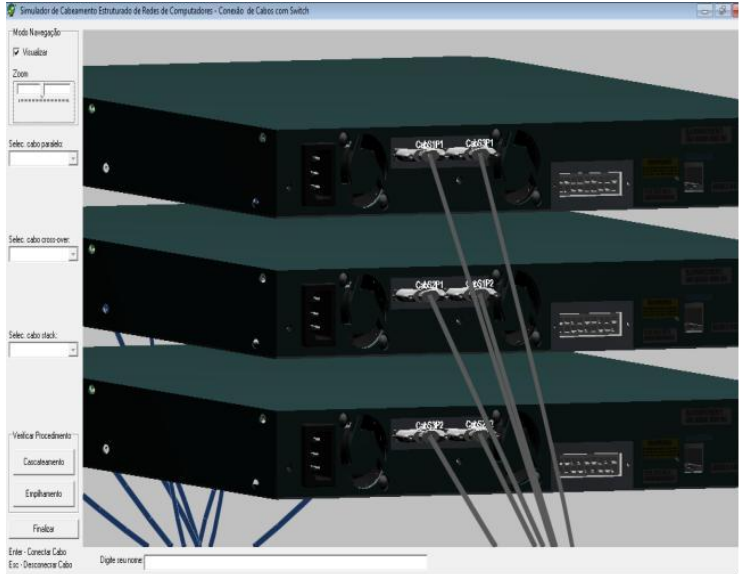

d) Empilhamento de Switchs

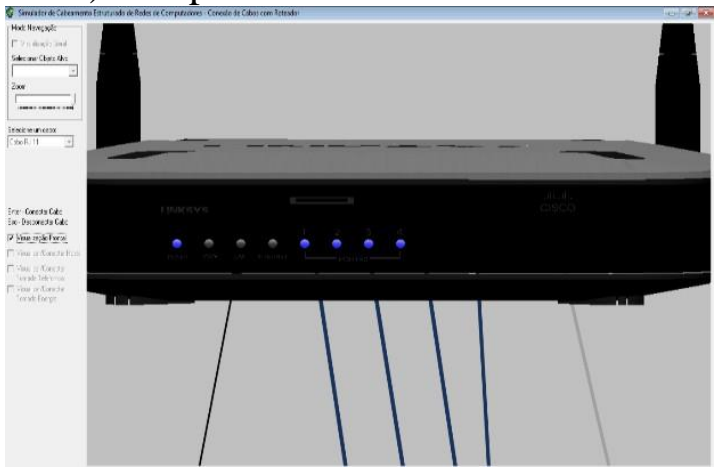

f) Reação eletrônica do roteador

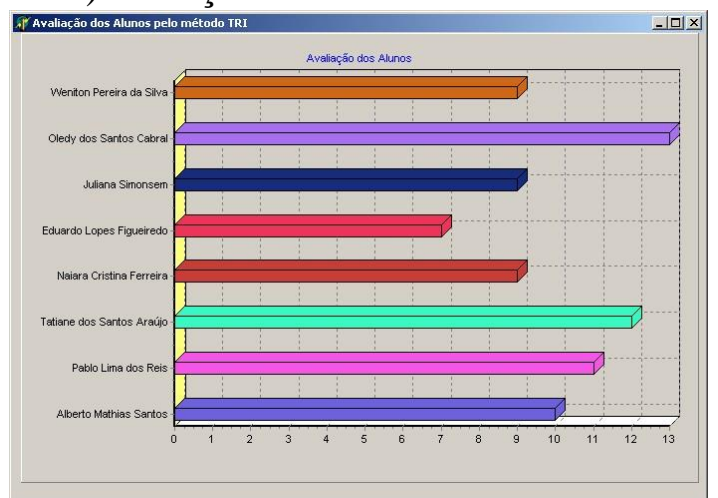

h) Verificação do desempenho

Figura 2. Telas do protótipo

A interação do usuário com o sistema é realizada parte utilizando comandos auxiliares, que basicamente definem as ações que o usuário irá tomar e a outra parte é através da movimentação dos objetos da cena utilizando o mouse e o teclado.

\section{Avaliação da Aprendizagem Imersiva}

Quanto a avaliação da aprendizagem imersiva promovida pelo Sistema foi estabelecido que a avaliação segue dois momentos distintos: o primeiro sobre o ambiente, com a avaliação dos requisitos de software voltado à área educacional[Souza et al., 2010] e elementos como a ergonomia, interface, interação humano-ambiente, entre outros [Pietruchinski et al., 2011]; e em segundo plano, a avaliação quanto ao processo de 
ensino e de aprendizagem, como estudos de estratégias e metodologias que envolvam mecanismos de interação e avaliação neste ambiente[Soares et. al. 2006].

A avaliação é uma etapa obrigatória do processo educacional, porém o centro desse processo certamente é a aprendizagem, envolvendo aspectos de interação entre os participantes (alunos e professores).

No processo de aprendizagem, é importante considerar inúmeras variáveis, uma delas é o perfil do aluno que se percebe pelas escolhas na forma de construir seus conhecimentos, por exemplo, um aluno pode realizar experiências no ambiente armazenando seus dados e conclusões num banco de dados particular, mas também compartilhar essas experiências com os outros participantes, assim como se envolver numa construção cooperativa de uma experiência coletiva, mas mantendo sempre sua individualidade.

Neste ambiente, o processo de avaliação, deve possibilitar a relação entre todos os elementos que fazem parte de um processo de ensino e de aprendizagem, incluindo objetivos (de professores e alunos) e conteúdo, pressupondo os elementos cognitivos (memória, inteligência, consciência), os elementos afetivos (atitudes, valores, criatividade) e os elementos psicológicos (vivência, sentimentos). Esses três elementos apontam para o planejamento de propostas educativas mais amplas e coerentes com o meio, acultura e a cognição.

\section{O uso do Simulador por alunos de um curso profissionalizante de redes de computadores}

De maneira a buscar um processo de melhoria contínua do simulador, foi realizada uma avaliação inicial com alunos de curso profissionalizante os quais responderam um questionário, empregando perfis aos usuários do sistema utilizado e também dimensionando uma possível evolução da prática de técnica de redes de computadores.

Previamente à avaliação, foram selecionados aleatoriamente 23 discentes, cursando o curso técnico de informática, e 12 alunos do projeto PRONATEC de Instalador e Reparador de Redes de Computadores. Esta seleção possibilitou a coleta de opiniões de diferentes públicos, relacionados aos discentes com diferentes níveis de conhecimento em prática de redes de computadores. Após a seleção dos participantes, um questionário inicial de múltipla escolha foi empregado, com o objetivo de se estabelecer o perfil de conhecimento de cada aluno.

É importante frisar que nenhum tipo de instrução formal relacionada a qualquer tópico de redes de computadores foi fornecida aos estudantes, antes ou durante a avaliação, sendo exibidas apenas explicações gerais sobre o funcionamento do simulador e seu objetivo.

Após a avaliação de conhecimento dos participantes, os mesmos foram apresentados ao software, realizando as primeiras interações com o instrutor mostrando como operar o simulador.

Para melhor acurácia do experimento, cada usuário realizou cada fase do processo 3 vezes, sendo que a cada nova fase questões relativas ao entendimento tanto do conteúdo quanto da forma de ação requerida com o dispositivo de rede são avaliados pelo

sistema. 


\section{Resultados}

De forma a elucidar o perfil do usuário do sistema deste experimento a Figura 3 evidencia que $60 \%$ do grupo é composto de pessoas leigas no assunto de instalação e reparo de redes de computadores, sendo este fator relevante para o uso do sistema, pois este grupo reflete se realmente houve uma eficiência da aplicação do simulador em sala de aula. Esta premissa é respondida pela análise da Figura 4. Na Figura 4 é evidenciado que $82 \%$ dos alunos após o uso do simulador conseguiram realizar os procedimentos de instalação e reparo de redes. E apenas $17 \%$ dos pesquisados tiveram insucesso no processo. Destes alunos foi observado que $95 \%$ souberam identificar a tarefa real e realizar a operação real a partir da simulação já realizada.

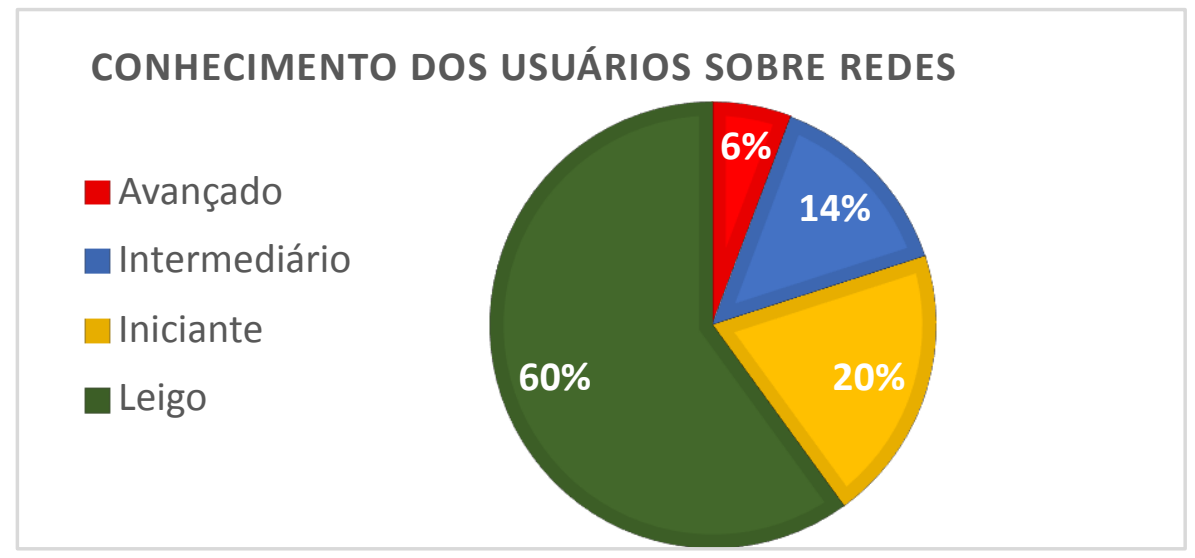

Figura 3. Identificação do perfil do aluno

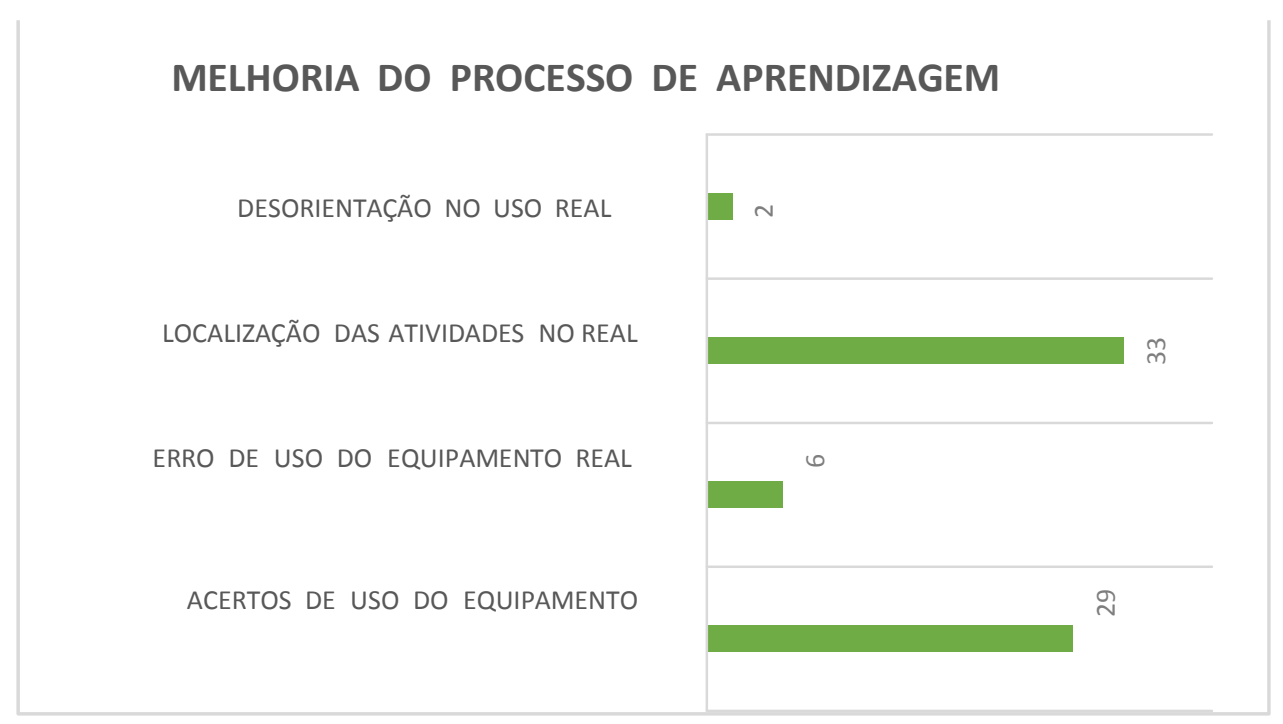

Figura 4. Análise dos erros de uso no processo de aprendizagem

No que tange aos aspectos do uso do software, nota-se que os alunos identificaram que o existe uma facilidade de uso na operação do simulador, todavia apenas 28 dos 35 alunos responderam que aprendeu algum conceito novo com o simulador, não se sabe se os usuários avançados e intermediários influenciaram a turma ou se realmente não houve mostra de novos conceitos apresentados aos alunos.

O que mais tem efeito de validade é que quase $90 \%$ dos alunos que usaram o simulador afirma que o sistema promoveu a prática dos conceitos demonstrados pelo professor 


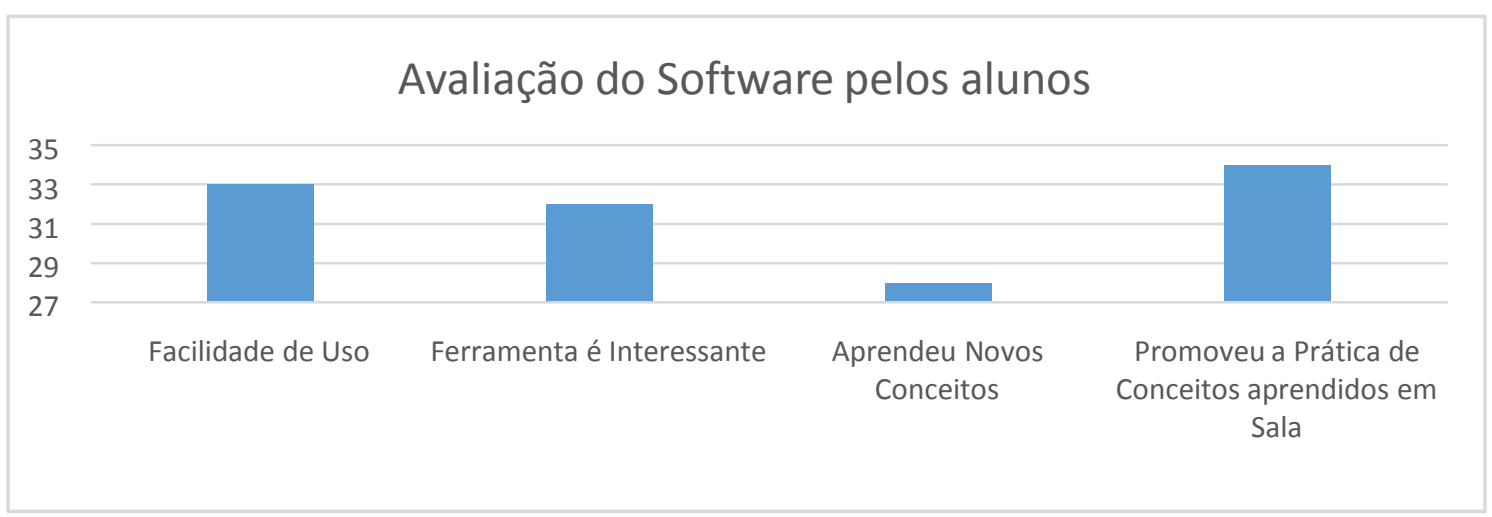

Figura 5. Telas do protótipo

\section{Contribuições e Considerações Finais}

Este trabalho abordou o projeto e o desenvolvimento de um software que usa RV para auxiliar atividades de instrução sobre a confecção e instalação de redes de computadores, foco da disciplina de redes de computadores no programa PRONATEC. Este simulador possibilitou uma interação dinâmica do usuário com o sistema por meio da interação e visualização mais próxima do real dos dispositivos sem a necessidade de usá-los fisicamente, o que favorece a preservação do equipamento e evita danos ao patrimônio público contido nas universidades e centros de ensino técnico e tecnológico. Em uma nova fase do experimento e do simulador estará sendo desenvolvida uma nova versão com acesso a dispositivos de interação gestual, com os quais se acreditam que poderão dar maior envolvimento no simulador. Ademais, outras atividades que comporão a nova versão dos simuladores será a "gamificação" de todo o processo.

\section{Referências}

Adlong, W.,Bedgood, Jr., D. R., Bishop, A. G., Dillon, K., Haig, T., Helliwell, S., Pettigrove, M., Prenzler, P. D., Robards, K. and Tuovinen, J. E. (2003) "On the path to improving our teaching - reflection on best practices in teaching chemistry". Proceeding softhe 2003 conference of the Higher Education Research and Development Society of Australasia.

Borin, J. F; Fonseca, N, L, S. (2008)“'Um módulo para simulação de redes WiMAX no simulador NS-2” . In: XXVIII Congresso da Sociedade Brasileira de Computação CSBC. Belém, 2008.

BRASIL MEC (2010) “Catálogo Nacional de Cursos de Técnicos”. Disponível em http://portal.mec.gov.br. Acesso em 10/09/2010.

BRASIL PRONATEC - Programa Nacional de Acesso ao Ensino Técnico e Emprego, Ministério da Educação e Cultura, acesso em 14/12/2013.

BRASIL. Constituição (2011). Decreto $n^{\circ}$ 7589, de 27 de janeiro de 2011. Institui A Rede E-tec Brasil. DF, 2011.

Cantú, E. (2005) Elementos para o fortalecimento da mediação docente na educação tecnológica aplicação no ensino - aprendizagem de redes de computadores. 2005. 176 p. Tese de Doutorado (Doutorado em Engenharia Elétrica). Universidade Federal de Santa Catarina. 
Damasceno, EF.; Lopes, LFB; Dias Jr, JB; Ramos, FM; Cardoso, A. (2008) "Um Estudo de Caso sobre a Engenharia de Sistemas Baseada em Componentes para Realidade Virtual", In: X Symposium of Virtual and Augmented Reality 2008, pp 167-170. João Pessoa-PB, SVR2008.

Ferreira, K. H., De Lima, R. W., Lima, M. V. D. A., \& Chaves, O. M. (2013) Laboratório Virtual para o Ensino de Redes de Computadores no Moodle. In Anais do Simpósio Brasileiro de Informática na Educação (Vol. 24, No. 1), 2013.

Filippo, D., Raposo, A., Endler, M. \&Fuks, H. (2007) “Ambientes Colaborativos de Realidade Virtual e Aumentada". in: Realidade Virtual e Aumentada - Conceitos, Projeto e Aplicações, Cláudio Kirner e Robson Siscoutto (eds), Editora SBCSociedade Brasileira de Computação, Porto Alegre, 2007.

Hounsell, M. S.; Silva, E. L.; Gasparini, I. (2008) “Análise Das Ênfases De Educação E Treinamento Em Ambientes Virtuais 3D.” Editora Bookmark, São Paulo - Brasil.

Jukemura, A., H. Nascimento, eF. Costa. (2006) "Exploração da Realidade Aumentada no Aprendizado e Gerenciamento de Redes de Computadores." WORKSHOP DE REALIDADE AUMENTADA, Rio de Janeiro. Proceedings: WRA. 2006.

Martins, A.R. (2008) “O Melhor do Computador". Nova Escola, A revista de quem educa. Ano XXIII. N 215. Página 84 e 85. Editora Abril. Setembro 2008.

Meiguins, B. S., Behrens, F. H., Meiguins, B. S., \& Ferreira, D. O. (2000) Tecnologia de Realidade Virtual para o Auxílio no Aprendizado em Sala de Aula para Circuitos Elétricos. XX SBC/VI WIE, Curitiba. 2000.

Nedic, Z.;Machotka, J.; Nafalski, A., (2003). "Remote laboratories versus virtual and real laboratories," Frontiers in Education 2003.

NETACAD (2014), Cisco Networking Academy. Disponível em:<https://www.netacad.com/pt/web/about-us/cisco-packet-tracer>. Acesso em: 14 Jan. 2014.

Oliveira, RV. (2006) "Qualificar para quê? Qualificação para quem? do global ao local: o que se espera da qualificação profissional hoje" /. São Paulo: Fundação Unitrabalho, 2006.

Pan, J. (2010) “A Survey of Network Simulation Tools: Current Status and Future Developments", Case Report, disponível em: http://www1.cse.wustl.edu/ jain/cse567-08/ftp/simtools/index.html\#OPNET

Pietruchinski, M. H., Coelho Neto, J. Malucelli, A., \& Reinehr, S. (2011). Os jogos educativos no contexto do SBIE: uma revisão sistemática de Literatura. In Anais do Simpósio Brasileiro de Informática na Educação, 2011.

Pinheiro, CDB. (2005) "LVR-Laboratório Virtual de Redes-Protótipo para Auxílio ao Aprendizado em Disciplinas de Redes de Computadores." Anais do Simpósio Brasileiro de Informática na Educação. 2005.

SAGSET, (2014) "S. Society for the Advancement of Games and Simulations in Education and Training, SAGSET Home page," 2014. [Online]. Available: www.sagset.org 
Shannon, R.E. (1998) "Introduction to the Art and Science of Simulation," in Proceedings of the 1998 Winter Simulation Conference, 1998, pp. 7-14.

Soares, J. M., Leite, F. D. R., Mattos, C. L. C., Barroso, G. C., Sampaio, R. F., \& Leão, R. P. (2006, January). Acesso Remoto e Compartilhamento de Simuladores através de Ambientes Virtuais de Aprendizagem. In Anais do Simpósio Brasileiro de Informática na Educação. 2006.

Souza, M. M., Resende, R. F., Prado, L. S., Fonseca, E. F., Carvalho, F. A., \& Rodrigues, A. D. (2010). SPARSE: Um Ambiente de Ensino e Aprendizado de Engenharia de Software Baseado em Jogos e Simulação. In Anais do Simpósio Brasileiro de Informática na 